\title{
Orientationsstruktur der Talanlagen in der Schweiz
}

\section{ABSTRACT \\ Orientation-pattern of the valley-trends in Switzerland}

The orientation of the valleys in Switzerland shows a remarkable symmetry. It is the aim of this paper to represent this symmetry in numerical terms and to seek a physical cause thereof. For this purpose, the valley directions have been «rectified» (i. e. straightened) by considering them as edges in a graph. In this fashion, the distribution of orientations can be represented numerically and, in consequence, can be analyzed statistically. It is shown that the preferred orientations are ENE and SSE, which is the same as the preferred orientations of the strikes of the rock-joints in the region. Inasmuch as the joints are thought to be caused by the neotectonic stress field, the coincidence of the orientations of joints and river valleys is a strong indication that the latter are determined by the same neotectonic stress field as well. Data from fault plane solutions of earthquakes support this contention.

\section{Einleitung}

Ein Blick auf eine Eisenbahnkarte der Schweiz läßt einen sofort eine gewisse Symmetrie erkennen: Zur Achse Basel-Luzern-Gotthard liegen symmetrisch die Linien Brugg-Sargans und Brugg-Bern, dazu noch die Linien Chur-Andermatt und Brig-Andermatt.

Die Bahnlinien folgen natürlich in erster Linie den Tälern. Die Symmetrie des Eisenbahnnetzes ist daher durch die Symmetrie der Talanlagen der Schweiz bestimmt, die systematisch etwa wie in Abb. 1 skizziert werden kann. Man kann sich fragen, ob die intuitiv erkennbare Regelmäßigkeit auch quantitativ erhärtet werden kann, und, falls dies der Fall ist, was der Grund dafür sein möge.

Wir wollen daher in der vorliegenden Arbeit die Orientationsstruktur der Talrichtungen erforschen. Hierzu ist es zuerst einmal nötig, die Talrichtungen numerisch festzulegen. Dann kann eine statistische Auswertung derselben in Angriff genommen werden, und schließlich können die erhaltenen Verteilungen mit denen der Orientierungen von Klüften und Erdbebenherdebenen verglichen werden. Es wird sich zeigen, daß eine Übereinstimmung besteht, so daß man fordern muß, daß die

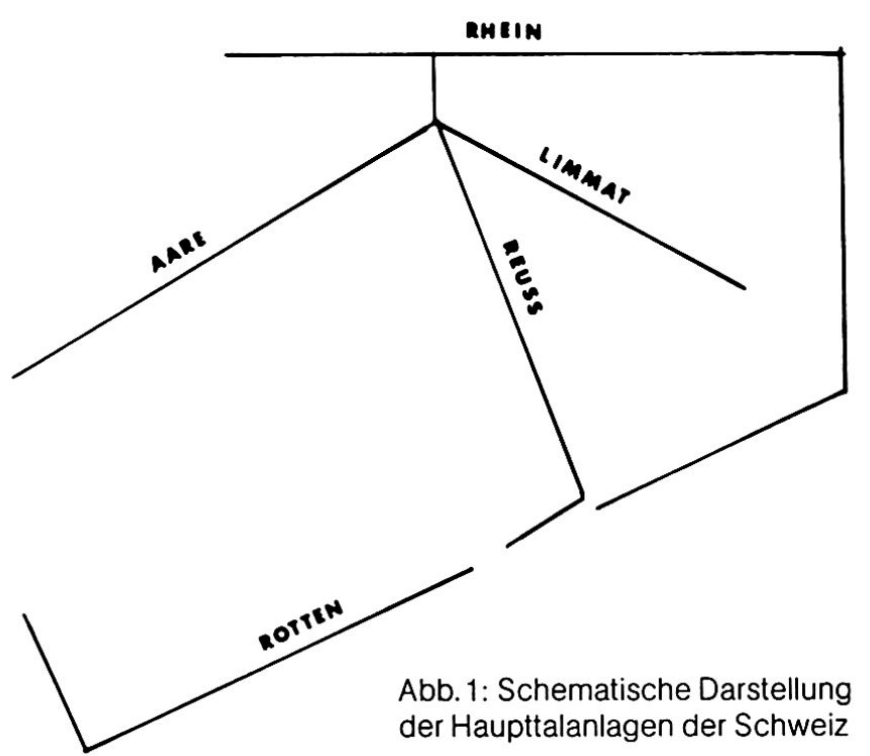

Orientierung der Talanlagen durch dieselbe Ursache wie diejenige der Kluftstellungen und Erdbebenherde, d. h. durch das neotektonische Spannungsfeld, erzeugt wird.

\section{Digitisierung von Talrichtungen}

Um die intuitiv gespürte Symmetrie der Schweiz quantitativ zu erfassen, ist es erst einmal notwendig, die Talrichtungen quantitativ festzulegen. Man wird sich dabei auf gewisse Gebiete beschränken müssen; als natürliche Abgrenzungen bieten sich hierbei für unsere Untersuchung das Einzugsgebiet des Rheins bis Basel und des Rotten bis Genf an. Wir haben daher die Flußläufe aus einer Karte (die «blauen Linien») nachgezeichnet und die oben beschriebenen Einzugsgebiete darauf eingetragen (Abb. 2).

Als nächstes Problem stellt sich jenes der Definition von Talrichtungen mit entsprechenden "Gewichten», damit statistische Untersuchungen gemacht werden können. Nun wird aber ein Flußlauf durch eine Schlangenlinie (engl. "wiggly line») repräsentiert und die Definition von Richtungen bietet recht grundsätzliche semantische Schwierigkeiten (Ghosh und Scheidegger, 1971). Je größer der Kartenmaßstab, desto mehr Un-

Prof. Dr. Adrian E. Scheidegger, Technische Universität Wien, Institut für Geophysik, Gußhausstraße 27-29, A-1040 Wien. 


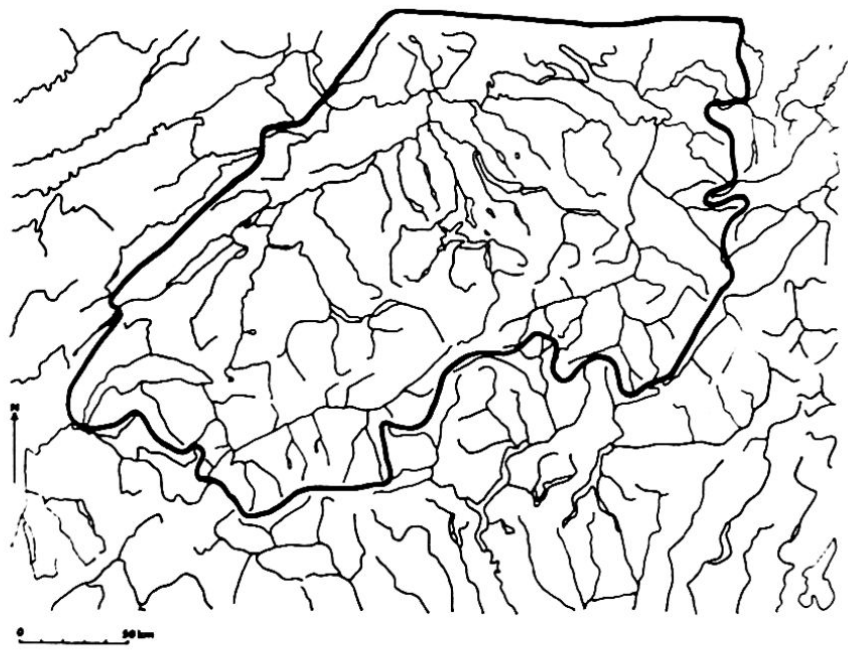

Abb. 2: Flußläufe und betrachtete Einzugsgebiete.

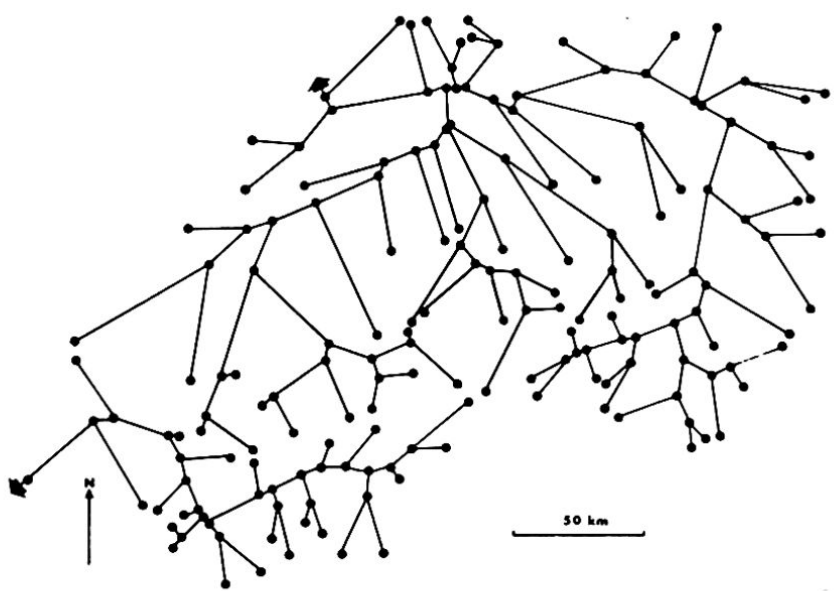

Abb. 3: Rektifizierter Graph der betrachteten Einzugsgebiete.

regelmäßigkeiten werden erkennbar, und man kann sich fragen, ob so eine Linie nicht überhaupt als «nichtdiffenzierbar» zu gelten habe. Ein Ausweg aus der Situation ergibt sich, wenn man ein Einzugsgebiet, wie es auf einer gegebenen Karte dargestellt ist, als Graphen betrachtet, in dem man die freien und inneren Vertices einfach durch gerade Kanten verbindet (Rektifizierung). Das ist natürlich ein etwas brutales Vorgehen, aber wenigstens eines, das von dem Individuum, das die Untersuchung durchführt, statistisch unbeeinflußt ist. Die Orientationsstruktur des Graphen ist dann einzig und allein durch die «blauen Linien» der vorliegenden Karte bestimmt. Die Rektifizierung des von uns betrachteten Gebietes der Schweiz nach obigem Schema ergibt das in Abb. 3 gezeigte Bild.

Nach der Rektifizierung der Talrichtungen ist es eine einfache Sache, deren Orientation zu messen; diese wird einfach als die Richtung des Streichens $(\mathrm{N} \rightarrow \mathrm{E})$ der Graphenkanten angezeigt. Als statistisches $\mathrm{Ge}$ wicht in einer Auswertung der Orientationsstruktur der Talanlagen kann die jeweilige Länge der Kanten dienen.

\section{Statistische Auswertung der Schweizer Talrichtungen}

Man kann nun daran gehen, die Orientationsstruktur der Schweizer Talrichtungen statistisch zu erfassen. Für jede Kante des Graphen in Fig. 3 wird die Richtung $(\mathrm{N} \rightarrow \mathrm{E})$, das «Gewicht» als ihre Länge in Einheiten von $1,5 \mathrm{~km}$ notiert. Für die Auswertung wird auf ganze Einheiten gerundet. Als erstes kann man dann ein polares Histogramm ( $10^{\circ}$-Intervalle) der gewichteten Talrichtungen zeichnen; es ist in Abb. 4 gezeigt.

Für die weitere Auswertung ist es günstiger, statt des Streichens der Kanten deren Polrichtungen (d. h. Nor-

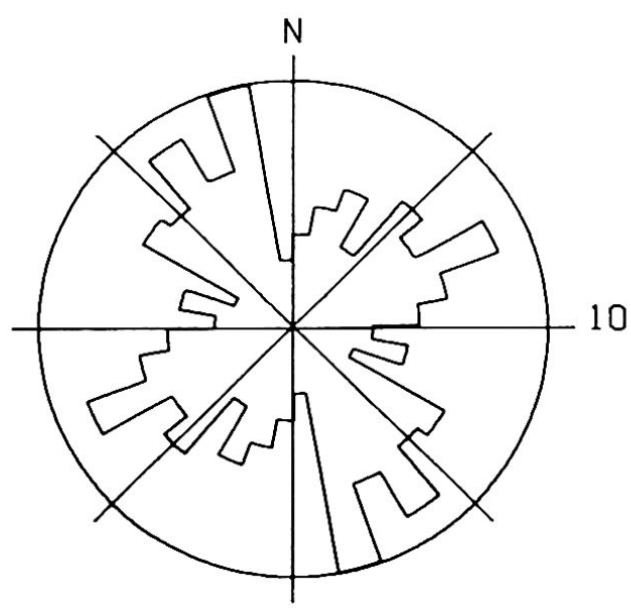

$\%$

SCHWEIZ TAELER STREICHEN

INTERVALL $\quad 10.0 \quad$ MAXIMUM $=10 \%$

$78 / 00510$ - 16.4001

Abb. 4: Histogramm ( $10^{\circ}$-Intervalle) des Streichens der Talrichtungen (Kreislinie entspricht $10 \%$ ).

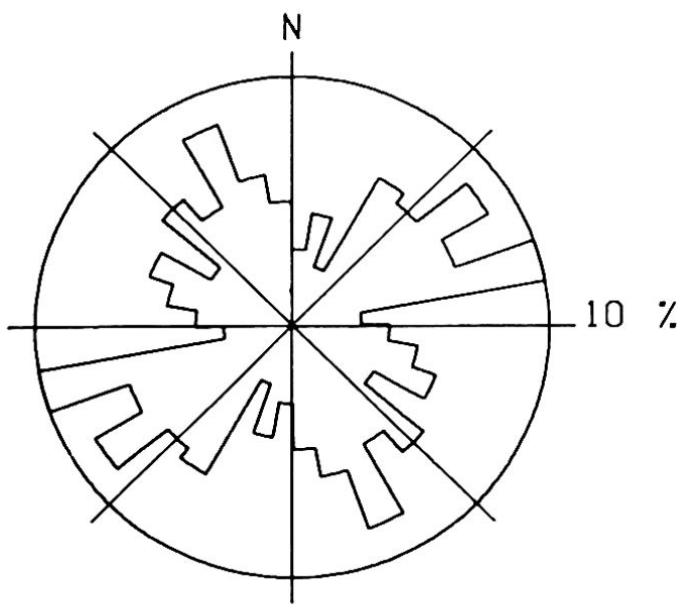

SCHWEIZ POLE VON TALRICHTUNGEN INTERVALL $\quad 10.0 \quad$ MAXIMUM $=10 \%$ $78 / 03 / 13-12.51 \mathrm{H}$

Abb. 5: Histogramm (10-Intervalle) der Pole der Talrichtungen (Kreislinie entspricht 10\%). 
male) zu betrachten, weil dies unserer üblichen Darstellung von Ebenendaten entspricht; - die Talrichtung ist dann quasi die Spur einer vertikal stehenden Ebene, deren Fallwinkel $90^{\circ}$ wäre. Bei dieser Betrachtungsweise wird das Histogramm von Fig. 4 einfach um $90^{\circ}$ gedreht (siehe Abb. 5).

Aus dem Histogramm der Klüftepole kann man sogleich zwei Maxima herauslesen. Diese liegen bei

Max. 1: $75^{\circ}$

Max. 2: $155^{\circ}\left(=335^{\circ}\right)$

Das Heraussuchen der Maxima nach obigem Schema entspricht dem Vorgehen in einer nichtparametrischen Statistik. Man kann sich vorstellen, daß es vorzuziehen wäre, die wahrscheinlichste Lage der Maxima für zwei überlagerte parametrische Verteilungen zu ermitteln. Wenn man als Grundverteilungen Verteilungen vom Typ A exp (-k $\left.\left.\cos ^{2} \eta\right)\right)$ ansetzt, kann man das Auswertungsprogramm von Kohlbeck und Scheidegger (1977) für Ebenen (Fallwinkel $=90^{\circ}$ ) verwenden. Man erhält dann

Max. 1: $55^{\circ} \pm 2^{\circ}$

Max. 2: $143^{\circ} \pm 2^{\circ}\left(=323^{\circ} \pm 2^{\circ}\right)$

wo die Fehlergrenzen dem 10\%-Vertrauensintervall entsprechen.

Die Diskrepanz zwischen den nichtparametrisch und parametrisch berechneten Maxima ergeben sich daraus, da $\beta$ die niederen Werte nicht symmetrisch um die Höchstwerte verteilt sind.

Die Existenz von zwei Maxima zeigt, daß die Orientationsstruktur der Einzugsgebiete von Rhein und Rotten tatsächlich sehr einfach ist: Es bestehen zwei bevorzugte Richtungen.

\section{Vergleich mit Kluftmessungen}

Um der Ursache der Orientationsstruktur der Talanlagen nachzugehen, empfiehlt es sich, vorerst zu untersuchen, ob eine Übereinstimmung mit den Kluftstellungen des betreffenden Gebietes besteht. Wir haben schon früher über Kluftstellungen berichtet (Scheidegger, 1977) und haben gezeigt, daß eine große Gleichförmigkeit der letzteren besteht. Seit der letzen Arbeit haben wir noch einige Regionen, die in die betrachteten Einzugsgebiete der Schweiz fallen, dazu genommen; die bevorzugten Kluftstellungen (Azimut der Fallrichtung $\mathrm{N} \rightarrow \mathrm{E}$ und Fallwinkel zur Horizontalen, in Altgrad) für alle in Frage kommenden Regionen sind in Tabelle 1 zusammengefaßt. Die mit \pm bezeichneten Werte beziehen sich auf die 10\%-Vertrauensgrenze. Wegen der groBen Uniformität der Kluftstellungen in der Schweiz ergaben sich gegenüber der früher publizierten Tabelle (Scheidegger, 1977) durch die neuen Messungen kaum Änderungen.

Zum Vergleich der Kluftstellungen mit Talrichtungen ist die frühere Darstellung der Kluftorientierungen durch Poldichtediagramme nicht sinnvoll. Es ist für den gegenwärtigen Zweck vorzuziehen, auch hier nur ein Histogramm für die Klüftepole zu zeichnen. Hierzu wurden die bevorzugten Kluftstellungen für jede der 20 «Regionen» als «Input» verwendet; das erhaltene Resultat ist in Abb. 6 dargestellt. Man erkennt sofort,

Tabelle 1 KlüfteSchweiz

\begin{tabular}{|c|c|c|c|c|}
\hline & Region & Zahl & Max. 1 & Max. 2 \\
\hline 1 & Panixerpaß & 106 & $244 \pm 9 / 83 \pm 8$ & $344 \pm 9 / 84 \pm 8$ \\
\hline 2 & Amden & 320 & $57 \pm 4 / 90 \pm 4$ & $330 \pm 9 / 87 \pm 8$ \\
\hline 3 & Ziegelbrücke & 65 & $60 \pm 11 / 89 \pm 10$ & $156 \pm 11 / 84 \pm 10$ \\
\hline 4 & Braunwald & 85 & $244 \pm 8 / 89 \pm 7$ & $328 \pm 10 / 84 \pm 9$ \\
\hline 5 & Säntis & 75 & $240 \pm 12 / 85 \pm 10$ & $151 \pm 20 / 85 \pm 19$ \\
\hline 6 & Surenenpaß & 85 & $56 \pm 6 / 88 \pm 6$ & $336 \pm 11 / 88 \pm 10$ \\
\hline 7 & Mythen & 98 & $26 \pm 19 / 83 \pm 13$ & $98 \pm 14 / 86 \pm 10$ \\
\hline 8 & Sihltalschluß & 42 & $105 \pm 11 / 73 \pm 10$ & $196 \pm 5 / 89 \pm 5$ \\
\hline 9 & Einsiedeln & 85 & $76 \pm 13 / 86 \pm 10$ & $342 \pm 26 / 90 \pm 19$ \\
\hline 10 & Sattel-Schwyz & 128 & $257 \pm 15 / 85 \pm 11$ & $337 \pm 18 / 82 \pm 16$ \\
\hline 11 & Roßberg & 95 & $259 \pm 11 / 84 \pm 10$ & $355 \pm 15 / 70 \pm 12$ \\
\hline 12 & Oberägeri & 54 & $63 \pm 13 / 88 \pm 12$ & $326 \pm 14 / 84 \pm 13$ \\
\hline 13 & Wädenswil & 70 & $64 \pm 13 / 89 \pm 10$ & $345 \pm 20 / 88 \pm 14$ \\
\hline 14 & Zürich & 42 & $256 \pm 12 / 89 \pm 11$ & $170 \pm 14 / 87 \pm 12$ \\
\hline 15 & Lenzburg & 45 & $249 \pm 11 / 89 \pm 11$ & $346 \pm 9 / 87 \pm 9$ \\
\hline 16 & Schinznach-Effingen & 82 & $282 \pm 14 / 86 \pm 12$ & $14 \pm 13 / 74 \pm 10$ \\
\hline 17 & Mumpfer Fluh & 65 & $290 \pm 12 / 90 \pm 9$ & $25 \pm 16 / 88 \pm 12$ \\
\hline 18 & Dietwil & 17 & $197 \pm 33 / 84 \pm 24$ & $120 \pm 23 / 83 \pm 18$ \\
\hline 19 & Kandertal & 140 & $89 \pm 14 / 86 \pm 10$ & $180 \pm 28 / 84 \pm 15$ \\
\hline 20 & Leukerbad & 200 & $51 \pm 4 / 85 \pm 4$ & $143 \pm 7 / 79 \pm 7$ \\
\hline
\end{tabular}




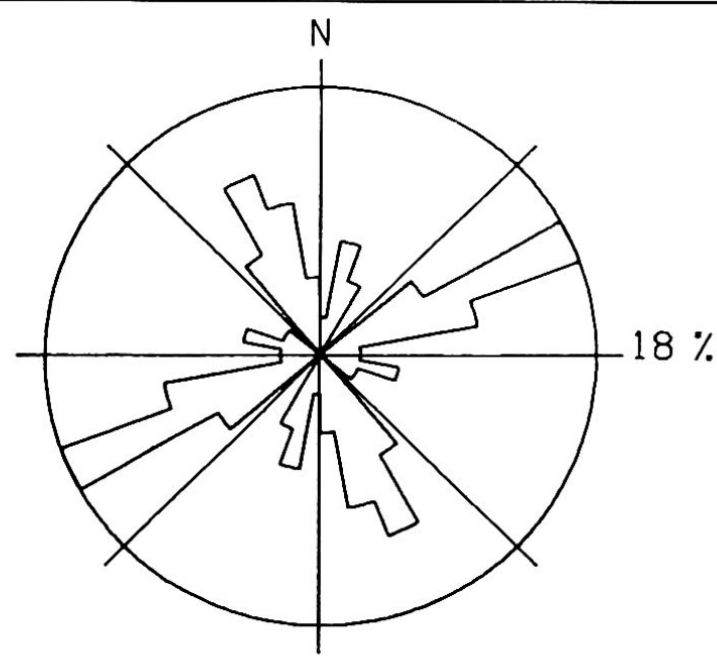

SCHWEIZ KLUEFTEPOLE

INTERVALL $\quad 10.0 \quad$ MAXIMUM $=18 \%$

Abb. 6: Histogramm ( $10^{\circ}$-Intervalle) der Klüftepole der Schweiz (Kreislinie entspricht 18\%).

daß es zwei Maxima gibt, nämlich

Max. 1: $65^{\circ}$

Max. 2: $155^{\circ}$

Man sieht, daß sich eine sehr gute Übereinstimmung der obigen Werte mit den nichtparametrisch bestimmten Maxima der Talorientierungen ergibt: Ein Maximum ist identisch, das zweite ist um nur eine Intervalleinheit $\left(10^{\circ}\right)$ verschoben.

Man kann auch bei den Kluftstellungen eine parametrisch-statistische Auswertung unternehmen. In diesem Falle werden zwei Dimroth-Watson-Verteilungen auf der Kugel angenommen und deren wahrscheinlichste Lagen berechnet. Wiederum werden die in jeder «Region» bevorzugten Kluftstellungen (Max. 1 und Max. 2 von Tab. 1) als Grunddaten («Input») verwendet und die bestpassenden Lagen der Maxima der Verteilungen parametrisch berechnet (Methode von Kohlbeck und Scheidegger, 1977). Man erhält

Max. 1: $74^{\circ} \pm 13^{\circ} / 90^{\circ} \pm 11^{\circ}$

Max. 2: $346^{\circ} \pm 15^{\circ} / 88^{\circ} \pm 12^{\circ}$

Man ersieht, daß sich hier eine gewisse Diskrepanz zwischen den parametrisch berechneten Maxima der Talrichtungen und der Kluftstellungen ergibt. Der Grund dafür liegt darin, daß die Verteilung der Daten außerhalb des Maximalbereiches in den beiden Fällen in etwas anderer Weise asymmetrisch ist. Im Rahmen der Genauigkeit der Messungen, die höchstens $5^{\circ}$ beträgt, und im Hinblick auf die statistisch ermittelten $10 \%$-Vertrauensgrenzen von $13^{\circ}-15^{\circ}$ kann man von einer guten Übereinstimmung der Tal- und Kluftrichtungen sprechen.

\section{Vergleich mit Erdbebenherdebenen}

Es ist bekannt, daß man für viele Erdbeben den Herdmechanismus bestimmen kann. Es erhebt sich die Frage, ob zwischen den Herdmechanismen von Erdbeben, deren Epizentren in dem von uns betrachteten Gebiet lagen, und den Talanlagen eine Beziehung herrscht.

$\mathrm{Zu}$ diesem Zweck wurden die für das in Frage kommende Gebiet publizierten Herdmechanismen gesammelt. Die meisten der Herdlösungen sind einer Schrift von Pavoni (1976) entnommen, der schon einen Kata$\log$ der Schweizer Herdmechanismen erstellt hatte; die restlichen zwei entstammen einer Arbeit des Schreibenden (Scheidegger, 1967). Pavoni (1976) hat nur die größte Zug-(T) und Druck-(P) Richtung für jede Herdlösung angegeben; wir haben die Stellungen (Fallrichtung und Fallen) der zugehörigen Knotenebenen (Aund C-Ebene) daraus berechnet. Die für unsere Studie in Frage kommenden Herdlösungen sind in Tab. 2 aufgeführt.

Tabelle 2 Erdbeben Schweiz

\begin{tabular}{|c|c|c|c|c|c|c|c|c|}
\hline Bez. & Datum & Region & Epizenter & A & $\mathrm{C}$ & $\mathrm{P}$ & $\mathrm{T}$ & Ref. \\
\hline A & $61-04-28$ & Schopfheim & 47.7 N. $7.9 \mathrm{E}$ & $176 / 90$ & $266 / 90$ & $134 / 18$ & $38 / 18$ & Pavoni 1976 \\
\hline B & $64-02-17$ & Sarnen (Vorb.) & $46.9 \mathrm{~N}, 8.2 \mathrm{E}$ & $50 / 90$ & $140 / 90$ & $275 / 0$ & $5 / 0$ & Scheidegger 1967 \\
\hline $\mathrm{C}$ & $64-03-14$ & Sarnen & $46.9 \mathrm{~N}, 8.3 \mathrm{E}$ & $171 / 72$ & $278 / 49$ & $139 / 11$ & $37 / 44$ & Pavoni 1976 \\
\hline $\mathrm{D}$ & $64-05-28$ & Graubünden & $46.8 \mathrm{~N}, 9,0 \mathrm{E}$ & $221 / 90$ & $131 / 90$ & $86 / 0$ & $356 / 0$ & Scheidegger 1967 \\
\hline E & $71-09-29$ & Glarus & $47.1 \mathrm{~N}, 9.0 \mathrm{E}$ & $203 / 86$ & $293 / 86$ & $158 / 0$ & $68 / 6$ & Pavoni 1976 \\
\hline $\mathrm{F}$ & $73-07-09$ & Arosa & $46.8 \mathrm{~N}, 9.7 \mathrm{E}$ & $21 / 90$ & $291 / 86$ & $156 / 3$ & $66 / 3$ & Pavoni 1976 \\
\hline G & $74-01-19$ & Berner Oberland & $47.7 \mathrm{~N}, 7.5 \mathrm{E}$ & $259 / 90$ & $349 / 83$ & $125 / 5$ & $214 / 5$ & Pavoni 1976 \\
\hline $\mathrm{H}$ & $74-04-26$ & Berner Mittelland & $42.2 \mathrm{~N}, 7.9 \mathrm{E}$ & $134 / 40$ & $20 / 71$ & $354 / 18$ & $240 / 51$ & Pavoni 1976 \\
\hline I & $75-12-29$ & Glarus & $42.1 \mathrm{~N}, 9.2 \mathrm{E}$ & $85 / 81$ & $177 / 80$ & $311 / 14$ & $41 / 1$ & Pavoni 1976 \\
\hline $\mathrm{J}$ & $76-01-29$ & Wallis & $46.3 \mathrm{~N}, 7.5 \mathrm{E}$ & $343 / 61$ & $219 / 45$ & $111 / 58$ & $8 / 10$ & Pavoni 1976 \\
\hline K & $76-03-02$ & Bodensee I & $47.6 \mathrm{~N}, 9.4 \mathrm{E}$ & $121 / 90$ & $211 / 90$ & $346 / 0$ & $76 / 0$ & Pavoni 1976 \\
\hline L & $76-03-22$ & St. Blaise & $47.0 \mathrm{~N}, 7.0 \mathrm{E}$ & $193 / 90$ & $283 / 90$ & $148 / 0$ & $58 / 0$ & Pavoni 1976 \\
\hline M & $76-03-26$ & Bodensee II & $47.6 \mathrm{~N}, 9.5 \mathrm{E}$ & $213 / 90$ & $123 / 90$ & $348 / 0$ & $78 / 0$ & Pavoni 1976 \\
\hline
\end{tabular}




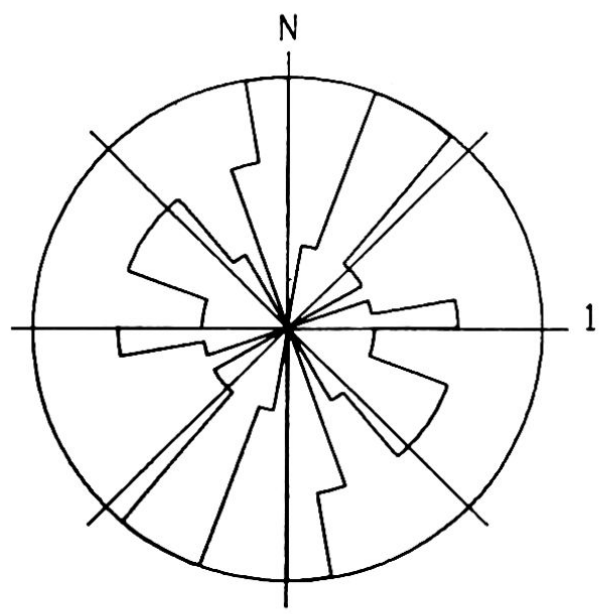

SCHWEIZ ERDBEBENHERDEBENEN

INTERVALL $\quad 10.0 \quad$ MAXIMUM $=12 \%$

$-78 / 04 / 03-14.004$

Abb. 7: Histogramm (10 $0^{\circ}$-Intervalle) der Erdbebenherdebenen der Schweiz (Kreislinie entspricht 12\%).

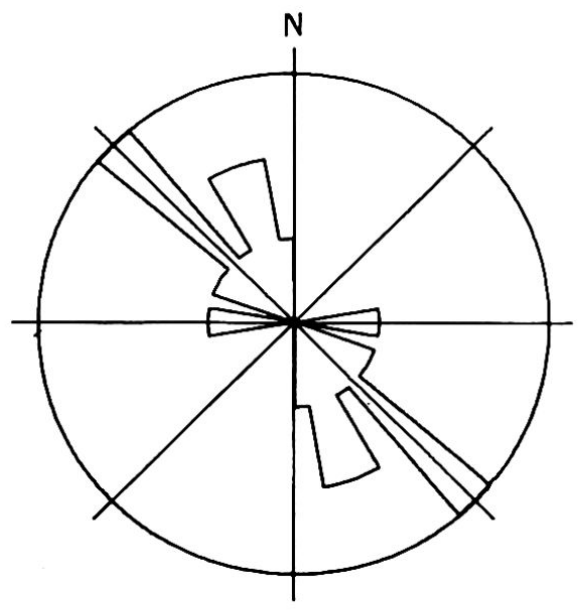

$24 \%$

\section{P-BEBEN SCHWEIZ}

\section{INTERVALL $\quad 10.0 \quad$ MAXIMUM $=24 \%$ \\ 78/04/91 - 11.30H}

Abb. 8: Histogramm (10-Intervalle) der P-Achsen der Erdbeben der Schweiz (Kreislinie entspricht 24\%).

Man kann sich nun fragen, ob zwischen diesen Knotenebenen und den Talrichtungen eine Beziehung bestehe. Dazu kann man wieder das Polarhistogramm für die Pole der Erdbebenherdebenen zeichnen (Abb. 7). Eine Betrachtung dieses Diagramms zeigt aber sofort, $\mathrm{da} ß$ man daraus keine sinnvollen Aussagen ablesen kann: Es gibt 4 Maxima, von denen je 2 benachbarte gleich stark sind. Die Schwierigkeit liegt offensichtlich darin, daß man die A- und C-Ebenen nicht einfach beliebig mitteln darf, da die Bewegungsrichtungen darauf jeweils verschieden sein können. Es ist daher vor-

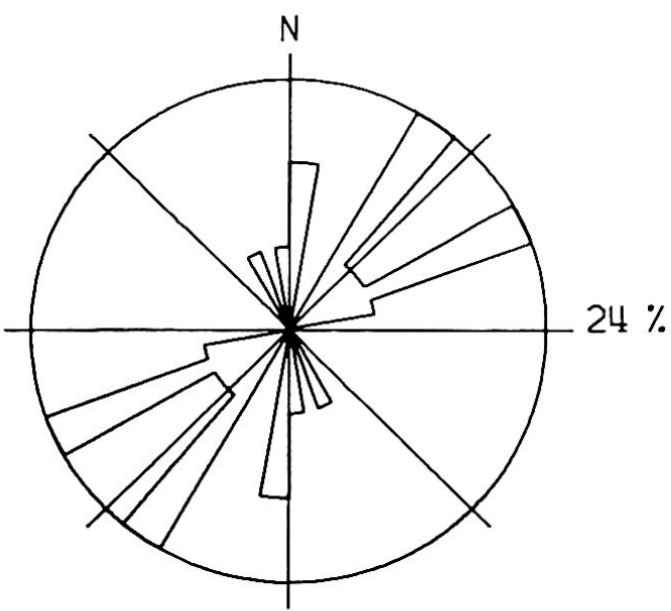

T-BEBEN SCHWEIZ

INTERVALL $\quad 10.0 \quad$ MAXIMUM $=24 \%$

$-78 / 04 / 07-11.024$

Abb. 9: Histogramm (10-Intervalle) der T-Achsen der Erdbeben der Schweiz (Kreislinie entspricht 24\%).

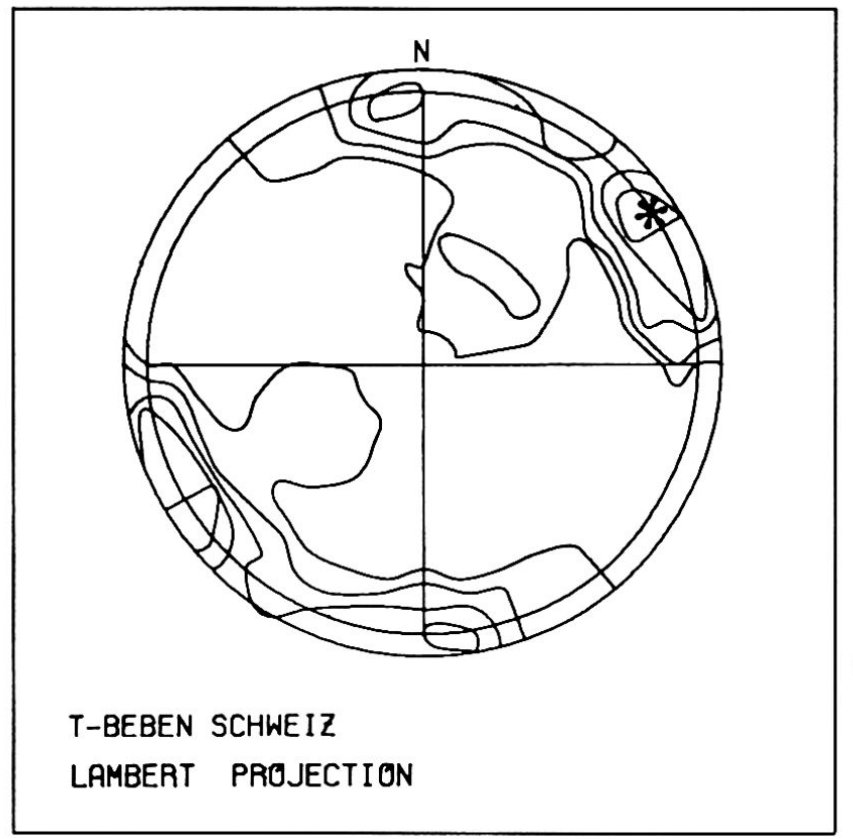

Abb. 10: Dichtediagramm (in Prozent) der Druckstoßpunkte der P-Achsen von Erdbeben der Schweiz durch die Einheitskugel (Lambert'sche Projektion).

zuziehen, die P- und T-Achsen der Erdbebenherdlösungen je für sich zu bearbeiten. Bei dieser Vorgangsweise kann man sicher sein, daß nur kinematisch gleichwertige Orientationen gemittelt werden.

Wir haben daher für die P- und T-Achsen zuerst einmal separate Polarhistogramme gezeichnet (Abb. 8 und 9). Es ist jedoch klar, daß auch hier keine eindeutigen Maxima festgestellt werden können. Ein Blick auf die numerischen Daten von Tab. 2 zeigt, daß manche der P- oder T-Achsen nicht sehr horizontal liegen, und daher ist die Vernachlässigung des Neigungswin- 


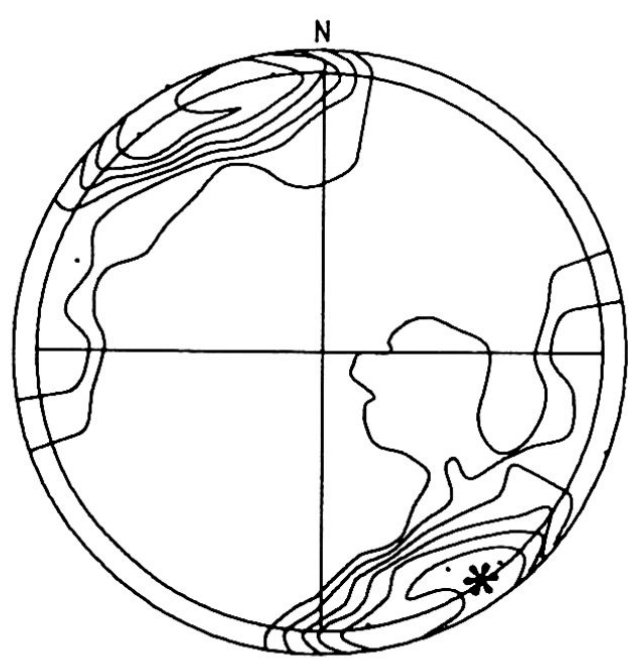

P-BEBEN SCHWEIZ

LAMBERT PROJECTION

Abb. 11: Dichtediagramm (in Prozent) der Durchstoßpunkte der T-Achsen von Erdbeben der Schweiz durch die Einheitskugel (Lambert'sche Projektion).

kels bei der Konstruktion der Polardiagramme ein zu grobes Verfahren. Es ist daher besser, Dichtediagramme der Achsen über die Einheitskugel zu zeichnen (Abb. 10,11). In diesen Diagrammen kann man dann eindeutig Maxima der Verteilungen erkennen, welche in den zwei Zeichnungen durch Sterne angedeutet sind. Diese Maxima liegen wie folgt

P: $145 / 0$

T: $55 / 0$

Diese Maxima liegen auch im rechten Winkel zueinander, so daß sie wirklichen Hauptspannungsachsen entsprechen können. Die winkelhalbierenden Ebenen würden dann den Kluft- und Talrichtungen entsprechen, sie haben die Orientierungen 90/90 und 180/90.

- Man kann nun auch versuchen, die Achsen parametrisch zu mitteln. Für jede Art Achse nimmt man die übliche $\cos ^{2}(\}$-Verteilung an; die Bestimmung der bestpassenden Achsenrichtung reduziert sich (bei je einer einzigen Verteilung) auf eine Eigenwertaufgabe (Fara und Scheidegger, 1963). Das Resultat ist

\section{P: $142 \pm 17 / 3 \pm 15$ \\ T: $37 \pm 24 / 6 \pm 22$}

Die großen $10 \%$-Vertrauensschranken spiegeln die große Streuung der Herdachsen wider (siehe Abb. 10, $11)$.

Die oben ermittelten bestpassenden Achsenrichtungen stehen nicht mehr orthogonal zueinander und können daher nicht die Hauptachsen eines Spannungstensors darstellen. Um eine Adjustierung vorzunehmen, bildet man die winkelhalbierenden Ebenen der Vektoren $\mathrm{P}$ und $\mathrm{T}$ und behandelt diese als "gemittelte» A- und C-Ebenen der betrachteten Gruppe von Erdbebenherden. Man erhält dann
A: $270 \pm 20 / 83 \pm 17$

C: $179 \pm 20 / 88 \pm 17$

wobei die Vertrauensschranken einfach die Mittelwerte der Schranken für $\mathrm{P}, \mathrm{T}$ sind. Aus diesen hypothetischen "gemittelten» Herdebenen kann man nun die $\mathrm{P}$ - und T-Richtung als Winkelhalbierende neu berechnen; diese Richtungen sind dann ex definitione aufeinander orthogonal. Man erhält

\section{P: $135 / 4$}

T: $44 / 6$

Wenn man die obigen Werte für A, C mit den Talrichtungen und Kluftorientierungen vergleicht, sieht man, daß die «gemittelten» Herdebenenorientierungen ungefähr um $15^{\circ}-25^{\circ}$ von den nichtparametrisch maximierten Tal- und Kluftorientierungen abweichen. Die Verteilung der Herdebenen streut aber in jedem Falle sehr stark (vgl. Abb. 7), so daß man die obigen Werte doch noch als eine Konkordanz, jedenfalls aber nicht als Beweis einer Diskordanz, einstufen kann.

\section{Geotektonische Betrachtungen}

Es verbleibt noch die Aufgabe, für die in den vorigen Abschnitten aufgezeigten Regularitäten eine physikalische Ursache zu ergründen. Hierzu wird man die gängige Auffassung herbeiziehen, daß die Klüfte Anderson'sche (1951) Bruchflächen in einem tektonischen Spannungszustand darstellen. Man kann dann die Hauptachsen dieses Spannungszustandes berechnen, wie das ja auch dem Argument in unseren früheren Arbeiten entspricht. Man erhält dann zwei Hauptachsenrichtungen $\left(\sigma_{1}, \sigma_{2}\right)$, die fast horizontal liegen; bei der parametrischen Auswertung liegen die Azimute bei $30^{\circ}$ und $120^{\circ}$, bei der nichtparametrischen bei $20^{\circ}$ und $110^{\circ}$.

Da die Talrichtungen den Kluftrichtungen durchaus entsprechen, kann man nun argumentieren, daß die Täler auch «Spalten» (Bruchflächen) im selben Spannungszustand darstellen. Es spricht somit alles dafür, da $\beta$ die Talanlagen durch das gleiche Spannungsfeld «vorgezeichnet» sind, welches auch die Klüfte erzeugt hat.

Ein Vergleich mit den Erdbebenherddaten führt zum gleichen Schluß. Auch hier ist es die gängige Auffassung, daß die gemittelten P- und T-Achsen der Erdbebenherde den neotektonischen Spannungshauptachsen entsprechen.

Zur besseren Veranschaulichung haben wir noch einmal alle errechneten Werte in einer Tabelle zusammengestellt (Tab. 3); die Werte beziehen sich auf die Polrichtungen der betrachteten Linearen oder Ebenen, wobei wir für das Weitere den Neigungswinkel außer acht gelassen haben. Zur leichteren Vergleichbarkeit sind die Azimute alle im Intervall $\left.\left(0^{\circ}<\psi\right) \leqq 180^{\circ}\right)$ genommen und in steigenden Werten für Max. 1, 2 geordnet. Die Azimute der entsprechenden Spannungshauptachsen sind auch aufgeführt. Eine Betrachtung 


\begin{tabular}{llllrr}
\hline & & Max. 1 & Max.2 & $\sigma 1$ & $\sigma 2$ \\
Täler & nicht-parametrisch & $75^{\circ}$ & $155^{\circ}$ & 25 & 115 \\
& parametrisch & $55^{\circ} \pm 2^{\circ}$ & $143^{\circ} \pm 2^{\circ}$ & 9 & 99 \\
Klüfte & nicht-parametrisch & $65^{\circ}$ & $155^{\circ}$ & 20 & 110 \\
& parametrisch & $74^{\circ} \pm 13^{\circ}$ & $166^{\circ} \pm 15^{\circ}$ & 30 & 120 \\
\multirow{3}{*}{ Erdbeben } & nicht-parametrisch & $90^{\circ}$ & $180^{\circ}$ & 55 & 145 \\
& parametrisch & $90^{\circ} \pm 20^{\circ}$ & $179^{\circ} \pm 20^{\circ}$ & 44 & 135 \\
\hline
\end{tabular}

dieser Tabelle läßt einen die starke Übereinstimmung der Spannungshauptrichtungen, die aus den Talrichtungen, Kluftstellungen und Herdlösungen erhalten wurden, erkennen. Der größte «Ausreißen» aller Werte ist der parametrisch aus den Talanlagen errechnete Wert. Wahrscheinlich sind doch bei den Talanlagen mehrere Systeme überlagert, so da $\beta$ das rezenteste, welches bei der nichtparametrischen Betrachtungsweise sofort evident ist, bei einer parametrischen Auswertung etwas verdeckt wird.

Man kann aus dem obigen also schließen, daß die Richtungen der Talanlagen der Schweiz durchaus den «Bruchflächen» des neotektonischen Spannungsfeldes entsprechen.

Die Schlußfolgerung, daß die Talanlagen der Schweiz äußerst rezent sind, sollte einen nicht überraschen. Die im in Frage stehenden Gebiet ermittelten Hebungsraten von der Größenordnung von $\mathrm{mm} / \mathrm{Jahr}$ (Gubler, 1976) bedeuten Vertikalbewegungen und entsprechende Abtragung von $\mathrm{km} /$ Millionen Jahre. Dies zeigt, $\mathrm{da} ß$ die gegenwärtige Topographie der Schweiz nicht älter als einige Millionen Jahre sein kann, also rezenten Ursprungs sein muß.

\section{Dank}

Der Schreibende wurde von Herrn Dr. Gerber in Schinznach auf das Problem der «Symmetrie» der Talanlagen in der Schweiz hingewiesen, wofür er ihm zu Dank verpflichtet ist. Stimulierend waren auch Diskussionen mit Herrn Dr. Pavoni in Zürich über die schweizerischen Erdbebenherdmechanismen. Die elektronische Datenverarbeitung wurde durch das Rechenzen- trum der Technischen Universität Wien, Abteilungen Digital- und Hybridrechenanlage, unentgeltlich durchgeführt, was hier gebührend verdankt werden soll.

\section{Literaturverzeichnis}

ANDERSON, E. M., 1951: Dynamics of faulting and dyke formation with applications to Britain. 2nd Ed., 206 p., London, Oliver \& Boyd.

FARA, H. D., SCHEIDEGGER, A. E., 1963: An eigenvalue method for the statistical evaluation of fault plane solutions of earthquakes. Bull. Seismol. Soc. Am. 53: 811-816 (1963).

GHOSH, A. K., SCHEIDEGGER, A. E., 1971: A study of natural wiggly lines in hydrology. J. Hydrol. 13: 101-126.

GUBLER, E., 1976: Beitrag des Landesnivellements zur Bestimmung vertikaler Krustenbewegungen in der Gotthard-Region. Schweiz. mineral. petrogr. Mitt. 56: 675-678.

KOHLBECK, F., SCHEIDEGGER, A. E., 1977: On the theory of the evaluation of joint orientation measurements. Rock Mech., 9: 9-25.

PAVONI, N., 1976: Herdmechanismen von Erdbeben und regionaltektonisches Spannungsfeld im Bereich der Geotraverse Basel-Chiasso. Schweiz. mineral. petrogr. Mitt. 56: 697-702 (1976).

SCHEIDEGGER, A. E., 1967: The tectonic stress in the vicinity of the Alps. Z. Geophys. 33(3): 167-181

SCHEIDEGGER, A.E., 1977: Kluftmessungen im Gelände und ihre Bedeutung für die Bestimmung des tektonischen Spannungsfeldes in der Schweiz. Geogr. Helv., 1977(3): 121-134. 